\title{
MIDTESOL 2013: “Engaging Learners, Building Community” Elizabeth Gould
}

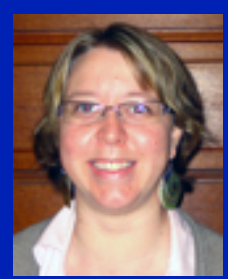

MIDTESOL is in Lawrence this year! This is a great way to put into practice the ideas discussed in this Supplement to ILI and you can consider it a test run for TESOL 2014. Also, the MIDTESOL conference is a wonderful opportunity to network with colleagues from the Midwest, especially, Iowa, Missouri, and other Kansas schools. Here are some details:

Place: $\quad$ Lawrence, KS

Date: $\quad$ October 12-13

Proposals: $\quad$ http://midtesol.org/midtesol/MIDTESOL\%202013\%20Call\%20for\%20Presentation\%20Proposals.pdf

Deadline: $\quad$ August 1, 2013

More Info: http://www.midtesol.org/midtesol/

\section{Volunteer at the Conference!}

If you would like to volunteer at the conference, please send me an e-mail at egould@ku.edu and I will add you to the list! There are a number of activities that will need to be completed before, during, and after the conference. This is a perfect opportunity for professional service. 


\section{Appendix}

Complete List of Interest Sections, Content Areas and Session Types for TESOL International Association ${ }^{5}$

\begin{tabular}{ll}
\hline Interest Sections \\
\hline - & Adult Education \\
- & Applied Linguistics \\
- & Compulingual Education \\
& Learning
\end{tabular}

- Elementary Education

- English as a Foreign Language

- English for Specific Purposes

- Higher Education

- Intensive English Programs

- Intercultural Communication

- International Teaching Assistants

- Materials Writers

- Nonnative English Speakers in TESOL

- Program Administration

- Refugee Concerns

- Secondary Schools

- Second Language Writing

- Speech, Pronunciation, and Listening

- Social Responsibility

- Teacher Education

- $\quad$ Video and Digital Media

Content Areas

- Accreditation

- Adult Education

- Applied Linguistics

- Assessment and Testing

- Bilingual Education

- Classroom management

- Community College

- Computer-Assisted Language Learning

- Content-based Language Instruction

- Curriculum, Materials Development

- Discourse, Pragmatics

- Elementary School/Primary School

- English as a Foreign Language

- English for Specific Purposes

- Grammar

- High School/Secondary Education

- Higher Education

- Integrated Skills

- Intensive English Programs

- Intercultural Communication

- International Teaching Assistants

- Language Policy and Planning

- Leadership

- Learning Disabilities

- Literature, Arts, Media

- Materials Writers

- Methodology

- Middle School/Preparatory Education

- Nonnative English Speakers in TESOL Types of Sessions

- Academic Session

- Colloquium

- Discussion Group

- Exhibitor Session

- Forum Session

- Intersection

- Invited Speaker

- Practice-oriented

- Research-Oriented

- Roundtable Discussion

- $\quad$ Teaching Tip

- TESOL in Focus

- Ticketed Event

- Workshop 
- Other

- Personal Development

- Program Administration

- Project-, Task-based Teaching and Learning

- Reading, Literacy

- Refugee Concerns

- Research and/or Research Methodology

- Second Language Acquisition

- Second Language Writing

- Social Responsibility

- Sociolinguistics, Culture

- Sociopolitical Concerns

- Speaking, Pronunciation, Phonology, Listening

- Specific Language Groups (Latino, Hmong, etc.)

- $\quad$ Speech, Pronunciation, Phonology, and Listening

- Standards

- Teacher Education

- Technology in Education

- Video and Digital Media

- Vocabulary, Lexicon

- Vocational, Workplace, Business English

- World Englishes

- Writing, Composition

- No Content Area 